\title{
Development of Polarization Interferometer Based on Fourier Transform Spectroscopy for Thomson Scattering Diagnostics
}

\author{
T. HATAE, J. HOWARD ${ }^{1)}$, Y. HIRANO ${ }^{2)}$, O. NAITO, M. NAKATSUKA ${ }^{3)}$ and H. YOSHIDA ${ }^{3)}$ \\ Fusion Research and Development Directorate, Japan Atomic Energy Agency, Naka 311-0193, Japan \\ 1) Plasma Research Laboratory, The Australian National University, Canberra ACT 0200 Australia \\ ${ }^{2)}$ National Institute of Advanced Industrial Science and Technology, Tsukuba 305-8568, Japan \\ ${ }^{3)}$ Institute of Laser Engineering, Osaka University, Suita 565-0871, Japan
}

(Received 11 December 2006 / Accepted 24 April 2007)

\begin{abstract}
A high-throughput polarization interferometer is being developed to demonstrate for the first time the utility of Fourier transform spectroscopy for Thomson scattering diagnostics of high temperature plasma. Target $T_{\mathrm{e}}$ and $n_{\mathrm{e}}$ ranges for the prototype polarization interferometer are $<1 \mathrm{keV}$ and $>5 \times 10^{18} \mathrm{~m}^{-3}$, respectively. This paper describes the design of the polarization interferometer and the results of initial tests.
\end{abstract}

(C) 2007 The Japan Society of Plasma Science and Nuclear Fusion Research

Keywords: Thomson scattering, Fourier transform spectroscopy, polarization interferometer, JT-60U, TPE-RX

DOI: $10.1585 /$ pfr.2.S1026

\section{Introduction}

Incoherent Thomson scattering [1] that can measure electron temperature $T_{e}$ and density $n_{e}$ profiles simultaneously is a standard diagnostic in magnetic confinement experiments. Usually grating spectrometers or interference filter polychromators have been used to analyze the scattered spectrum. Though these are established methods, the throughput decreases when there are many wavelength channels, while the relative calibration between wavelength channels is necessary.

It is well known that Fourier transform spectrometers [2] offer some potential advantages over dispersive systems. Fourier transform spectroscopy is a measurement technique whereby spectra are collected based on measurements of the temporal coherence of a radiative source, using time-domain measurements of the electromagnetic radiation or other type of radiation. In general, the main advantages of the Fourier spectroscopy are as follows. (1) The Fourier transform spectrometer (i.e. interferometer to measure the temporal coherence) can use the optical system of the high throughput (Jacquinot advantage), (2) Since the Fourier transform spectrometer observe the whole wavelength area at the same time, the $\mathrm{S} / \mathrm{N}$ ratio is improved (Fellgett advantage). More importantly, for this application, the method can be implemented in a simple and compact, high-throughput system. Optical coherence techniques have been applied successfully for plasma spectroscopy. In particular, high-throughput, wide field-of view polarization interferometers have been used for Doppler imaging of ion temperature in the $\mathrm{H}-1$ heliac [3-5]. These modulated or static coherence imaging systems monitor the complex coherence (fringe visibility and phase) of an

author'se-mail: hatae.takaki@jaea.go.jp isolated spectral line at one or more optical delays. A method based on measurement of the optical coherence of scattered radiation at a fixed optical delay has also been proposed for incoherent Thomson scattering [6]. However, this method has not been demonstrated to date.

To demonstrate this method, we are developing a Fourier filter for Thomson scattering based on a fixed delay polarization interferometer. Proof-of-principle tests will be carried out in TPE-RX reversed-field pinch (RFP) machine (Major radius $1.72 \mathrm{~m}$, Minor radius $0.45 \mathrm{~m}$, Plasma current $0.5 \mathrm{MA}, T_{e}(0) \sim 1 \mathrm{keV}$, pulse duration $\left.100 \mathrm{~ms}\right)$ [7], using the existing single channel YAG laser Thomson scattering system.

This paper describes the design of a prototype polarization interferometer and results of initial tests.

\section{Polarization Interferometer Based on Fourier Transform Spectroscopy}

The polarization interferometer is composed of a birefringent plate of fixed optical delay sandwiched between polarizers. When the light is observed through this interferometer, interference fringes appear at infinity due to Fresnel-Arago law. Thomson scattered radiation that traverses the first polarizer is incident on a birefringent plate whose fast axis is oriented at $45^{\circ}$ to the polarization direction. The plate splits the incident scattered scalar wave component, relatively delaying nominally equal amplitude components by time $\tau$ before they recombine at a final polarizer and are focused on to a detector. The orthogonally polarized outputs at the final polarizing splitter form complementary, or antiphase interferometric images of the input radiation. By suitably choosing the optical delay, these 


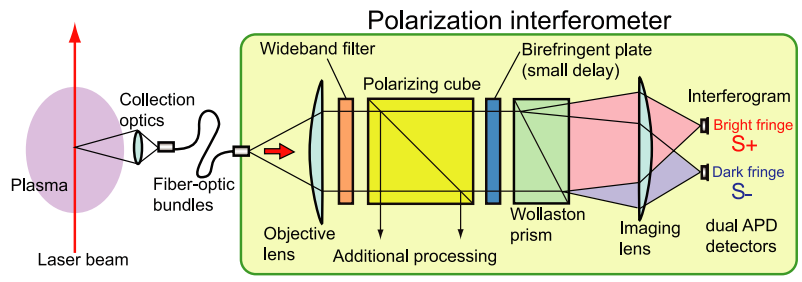

Fig. 1 Schematic of a polarization interferometer for Thomson scattering diagnostics.

independent outputs provide sufficient information to determine both the electron temperature and density.

A schematic of the polarization interferometer for Thomson scattering diagnostics is shown in Fig. 1. Scattered light is collected and introduced to the polarization interferometer through a fiber-optic bundle. This polarization interferometer consists an objective lens as the fiber coupling optics, a band pass filter, a polarizer, a birefringent plate which gives optical path delay, a Wollaston prism, an imaging optics to detector, and dual APD (silicon avalanche photodiode) detectors. Generally, the polarization interferometer is simple and low cost compared to the conventional polychromator.

The intensity of the transmitted light is proportional to

$$
S_{ \pm}(\tau)=\frac{I_{0}}{2}\{1 \pm \mathfrak{R}[\tilde{\gamma}(\tau)]\},
$$

where $I_{0}$ is the spectrally integrated irradiance, and $\tilde{\gamma}$ is the complex coherence. In general, the complex temporal coherence is related to the spectral distribution of the irradiance $I(v)$ through the Wiener-Khinchine theorem

$$
\tilde{\gamma}(\tau)=\frac{1}{I_{0}} \int_{-\infty}^{\infty} I(v) \exp (i 2 \pi v \tau) d v
$$

Here, $I_{0}$ is the spectrally integrated light intensity. The interferometric phase delay can be approximated by

$$
\phi=2 \pi v \tau(v) \approx \phi_{0}+\kappa \phi_{0} \xi
$$

where we have substituted $v=v_{0}(1+\xi)$, where $\xi=$ $\left(v-v_{0}\right) / v_{0}$ is a normalized frequency difference coordinate, $\phi_{0}=2 \pi v_{0} \tau_{0}$ : interferometric phase delay, where $\tau_{0}$ is the center-frequency time delay,

$$
\kappa=1+\left.\frac{\nu_{0}}{\tau_{0}} \frac{\partial \tau}{\partial v}\right|_{\nu_{0}}
$$

accounts for any optical frequency dispersion of the time delay. The interferogram is expressed following equation using Eq. (3) [6].

$$
S_{ \pm}\left(\phi_{0}\right)=\frac{I_{0}}{2}\left\{1 \pm \mathfrak{R}\left[\tilde{\gamma}\left(\hat{\phi}_{0}\right) \exp \left(i \phi_{0}\right)\right]\right\},
$$

where the optical coherence is given by

$$
\tilde{\gamma}(\hat{\phi})=\frac{1}{I_{0}} \int_{-\infty}^{\infty} I(\xi) \exp (i \hat{\phi} \xi) d \xi
$$

and it is convenient to introduce the group phase delay $\hat{\phi}_{0}=\kappa \phi_{0}$. We can evaluate the poralization interferometer response analytically in the low temperature case where the scattered spectrum is approximately Gaussian. For electron temperatures less than $1 \mathrm{keV}$, the spectral dependence of the scattered radiation is well approximated by

$$
I_{T}\left(\xi ; T_{e}\right)=I_{0}\left(\pi \xi_{t h}^{2}\right)^{-1 / 2} \exp \left(-\xi^{2} / \xi_{t h}^{2}\right)
$$

with

$$
\xi_{t h} \equiv 2 \sin (\theta / 2) v_{t h}
$$

and where $\theta$ is the angle between the incident and scattered wavevectors, $T_{e}$ is the electron temperature and $v_{t h}$ is the electron thermal speed normalized to the speed of light. Because the spectrum is a function only a single parameter, measurement of the coherence at a single fixed delay offset $\phi_{0}$ is sufficient to obtain $T_{e}$.

The normal-incidence time delay introduced by a birefringent crystal plate of thickness $L$ and birefringence $B$ is $\tau_{0}=L B / c$. For radiation of centre frequency $v_{0}$, the interferometer signal at either of the final polarizer ports is given by

$$
S_{ \pm}=\frac{I_{0}}{2}\left(1 \pm \zeta \cos \phi_{0}\right)
$$

where $\phi_{0}=2 \pi v_{0} \tau_{0}=2 \pi N$ is monochromatic birefringent phase delay and $N$ is the order of interference. The fringe visibility (fringe amplitude normalized to mean intensity) $\zeta=\zeta_{I} \zeta_{T}$ includes an instrumental component $\zeta_{I}$ due to the average of the birefringent plate delay over the angular extent of the source (analogous to familiar slit function for grating spectrometers) as well as the degradation $\zeta_{T}$ due to the finite source spectral width. The instrument function is determined via a suitable calibration procedure as discussed in section 4.

For a thermal distribution of $T_{e}<1 \mathrm{keV}$, the fringe visibility associated with the Thomson scattered light takes the simple from

$$
\zeta_{T}\left(\phi_{0}\right)=\exp \left[-\widehat{\phi}_{0}^{2} \sin ^{2}(\theta / 2) v_{t h}^{2}\right]=\exp \left(-T_{e} / T_{C}\right)
$$

where $T_{C}$ is a 'characteristic temperature' set by the waveplate delay and the scattering angle

$$
k T_{C}=\frac{1}{2} m_{e} c^{2} /\left[\hat{\phi}_{0} \sin (\theta / 2)\right]^{2} .
$$

Optimum sensitivity to temperature variation is obtained when the optical delay is chosen such that

$T_{e} \sim T_{C}$, or $\Delta v / v_{0} \sim 1 / N$, where $\Delta v / v_{0}$ is the Thomson spectral bandwidth [4].

For low temperatures, the variation of fringe visibility with temperature is best measured by setting $\phi_{0} / 2 \pi=M / 2$ where $M$ represents an integer number of half waves. A quartz waveplate will be used as the birefringent plate in 

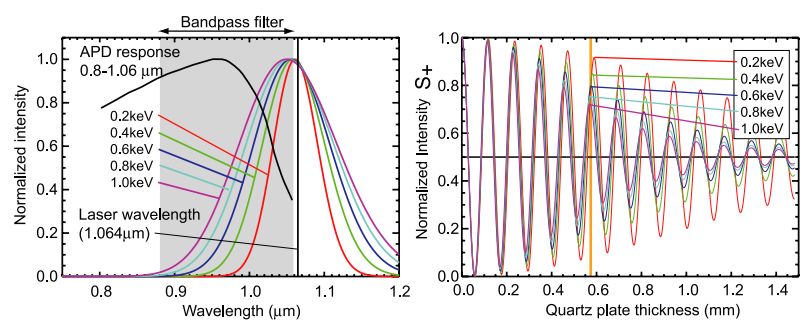

Fig. 2 Left: Thomson scattered spectra for temperatures in the range of $0.2-1.0 \mathrm{keV}$. Right: The calculated interferograms $\mathrm{S}_{+}$.

this design. The interferogram calculations take into account the wavelength-dependence of quartz birefringence and, for now, assume the spectrum to be ideally passed by the wideband filter. At a zero crossing, the scattered signals at the complementary output ports are

$$
S_{ \pm}=\frac{I_{0}}{2}\left[1 \pm \zeta_{T}\right],
$$

where $\zeta_{T}\left(\phi_{0}\right)$ is the Thomson spectrum fringe visibility at delay $\phi_{0}=M \pi$. The signals derived from both the orthogonally polarized light components produced by the final Wollaston prism are then sufficient to determine the total scattered power and the visibility degradation due to the Doppler broadening:

$$
\zeta_{T}=\frac{S_{+}-S_{-}}{S_{+}+S_{-}} .
$$

\section{Design of Polarization Interferome- ter for Proof-of-Principle Tests}

Proof-of-principle tests will be carried out in TPE-RX using the existing YAG laser Thomson scattering system [7]. Therefore, parameters for design of a prototype polarization interferometer are fixed as follows: $T_{e} \leq 1 \mathrm{keV}$, $n_{e} \geq 5 \times 10^{19} \mathrm{~m}^{-3}$, scattering angle $90^{\circ}$, YAG laser wavelength $1064 \mathrm{~nm}$. Thomson spectra and their associated interferograms for $T_{e}=0.2,0.4,0.6,0.8,1.0 \mathrm{keV}$ are shown in Fig. 2. To reject stray light by the YAG laser and reduce plasma background light, the wideband filter is placed at the entrance of the polarization interferometer. Wavelength range of the wideband filter will be $880-1060 \mathrm{~nm}$, with blocking ratio $10^{-6}$. Variations of fringe visibility $\zeta_{T}$ with electron temperature for the quartz plate thickness $=0.545$, $0.550,0.555 \mathrm{~mm}$ are shown in Fig. 3. Based on these calculations, a thickness of $0.555 \mathrm{~mm}$ will deliver a sensitive variation of fringe visibility with electron temperature as shown in Fig. 3.

\section{Calibration Methods}

The interferogram depends on both the nature of the scattered spectrum $I(v)$ as well as the overall system complex spectral response $R(v)$ which includes the angular dependence of the time delay (generally a small effect) as

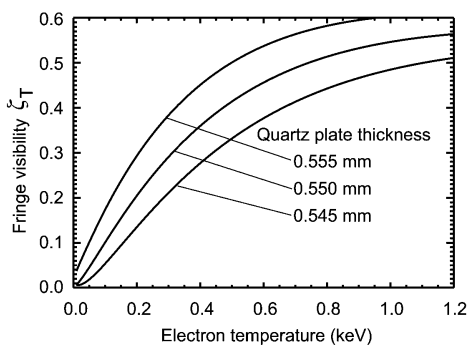

Fig. 3 Variations of fringe visibility $\zeta_{T}$ with temperature for three quartz plate thicknesses $(0.545,0.550,0.555 \mathrm{~mm})$.

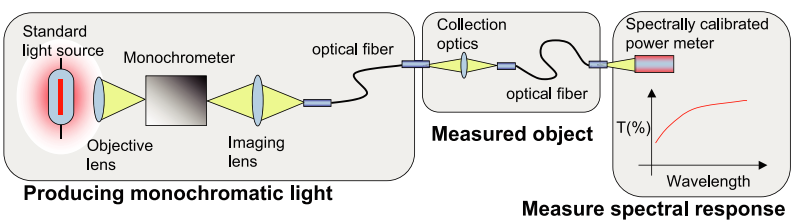

Fig. 4 Schematic of spectral transmissivity measurement.

well as the spectral response of the collection and processing optics and detection system. In this case we write the normalized fringe amplitude as $\zeta=\mathfrak{R}(\tilde{\gamma})$ and complex coherence as

$$
\tilde{\gamma}=\frac{1}{I_{0}} \int_{0}^{\infty} R(v) I(v) d \lambda .
$$

One means to determine the unknown response $R(v)$ would be measure the variation of the normalized fringe amplitude $\zeta_{B B}=\mathfrak{R}\left(\tilde{\gamma}_{B B}\right)$ for blackbody radiation as a function of the source temperature. For a given temperature $T_{j}$, Eq. (8) can be discretized in term of the unknown spectral response coefficients $R_{i} \equiv R\left(v_{i}\right)$ as

$$
\zeta_{B B}\left(T_{j}\right)=\sum_{i}\left(I_{B B}\right)_{j i} R_{i}
$$

where $\left(I_{B B}\right)_{j i}$ is the blackbody spectral irradiance at temperature $T_{j}$ and optical frequency $v_{i}$. For a sufficient number of measurements, Eq. (9) can be inverted for the response vector $\left\{R_{i}\right\}$. Actual measurement using the blackbody radiation source will be presented in the next section.

Spectral transmissivities between the plasma and the polarization interferometer should be measured as well. Because the Thomson scattered spectrum is distorted by spectral transmissivities of observation window, collection optics and optical fiber. Using these spectral transmissivities, spectra which enter the polarization interferometer can be corrected. The spectral transmissivity is measured as illustrated in Fig. 4. A monochromatic light is produced by a standard light source and monochromator. The power of transmitted light through the measured object is measured using a power meter which is calibrated spectrally. By comparing transmitted power with and without the measured object, the spectral transmissivity is obtained. Spectral sensitivity of the APD detector is also important 
for calibration. Generally, spectral sensitivity of the APD is used data provided by the maker. For the absolute density calibration, we consider Rayleigh scattering or Raman scattering calibration employing $\mathrm{N}_{2}$ gas [8].

\section{Initial Test using a Blackbody Ra- diation Source}

When the temperature of the blackbody is changed from $1273 \mathrm{~K}$ to $1773 \mathrm{~K}\left(1000-1500{ }^{\circ} \mathrm{C}\right)$, the spectrum changes as shown in Fig. 5. LAND R1500T as the blackbody radiation source is used for this test. The fringe visibility for the blackbody temperatures in this range, and in the bandpass $750 \mathrm{~nm}-1000 \mathrm{~nm}$, is calculated and plotted in Fig. 6 (solid line). To obtain a sensitive variation with temperature we used two crossed quartz waveplates having a measured effective thickness of $0.1293 \mathrm{~mm}$. Fringe visibilities for blackbody temperature in the range 1273-1773 K produced by the polarization interferometer are measured using two APD detectors, and the value of $\zeta$ is calculated using equation (7). The error bars are calculated taking into account the estimated $1 \mathrm{mV}$ uncertainty on the voltage levels. To fit ideal curve with experimental values, an arbitrary offset of value 0.11 in the visibility is required to be added to experimental values as shown in Fig. 6. The effective centre wavelength has to shift to the blue to account for this additional offset. Because most of the power from the blackbody radiation source is in the near infrared, the effective wavelength of the light in the APD passband is sensitive to the details of the APD responsivity. Moreover,

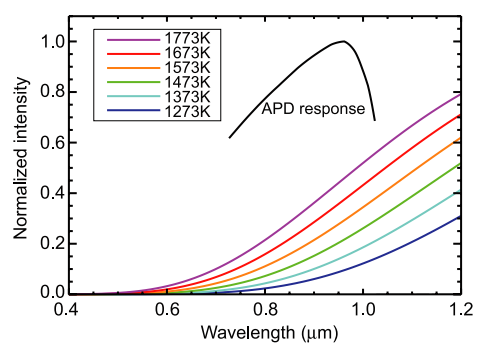

Fig. 5 Spectra of blackbody radiation source for temperatures in the range of $1273-1773 \mathrm{~K}$.

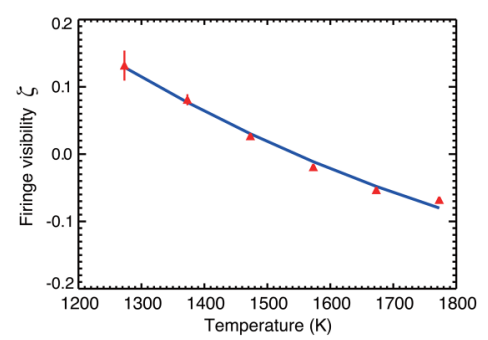

Fig. 6 Variations of fringe visibility $\zeta$ with temperature for a quartz plate thickness $(0.1293 \mathrm{~mm})$. The calculated fringe visibility (solid curve) is compared to experimental values (triangle). the interferometer instrumental fringe visibility degrades near $1000 \mathrm{~nm}$ due to the inefficiency of the first film polarizer. This will give rise to a blue bias, though we have not attempted to account for this numerically. The important point is that the magnitude of the change in fringe visibility agrees with the numerical calculation. This result confirms that, following suitable calibration, we will be able to sense visibility changes due to changes in the electron temperature.

\section{Discussion and Future Plan}

Based on results obtained using the prototype system, a high-throughput polarization interferometer has been designed for proof-of-principle tests. Target $T_{e}$ and $n_{e}$ ranges of the prototype polarization interferometer are $<1 \mathrm{keV}$ and $>5 \times 10^{18} \mathrm{~m}^{-3}$, respectively.

There are two remaining issues. The first issue is error analysis for $T_{e}$ and $n_{e}$. The second issue is an effect of background radiation (i.e. plasma light). These will be considered in a later paper.

Regarding future plans, proof-of-principle tests will be carry out in TPE-RX reversed-field pinch (RFP) machine in 2007. Incorporating improvements based on the results at TPE-RX, a new polarization interferometer will be designed for the JT-60U Thomson scattering system [9-11]. For this system we will consider (1) wider range of $T_{e}\left(T_{e}<40 \mathrm{keV}\right)$, (2) an imaging polarization interferometer for multi-spatial channel measurement (Maximum 20 spatial points), (3) using YAG laser $(50 \mathrm{~Hz}, 7.46 \mathrm{~J}$, $1064 \mathrm{~nm})$ [11] or ruby laser $(0.5 \mathrm{~Hz}, 10 \mathrm{~Hz}, 694.3 \mathrm{~nm})$ [12]. In the case of ruby laser, a high sensitivity CCD camera is available. After tests in TPE-RX and JT-60U, this diagnostic technique will be proposed for ITER and JT-60SA Thomson scattering system.

In this diagnostic technique, $T_{e}$ and $n_{e}$ can be directly determined from fringe visibility $\zeta_{T}$ measurement without iterative calculations. Since $T_{e}$ and $n_{e}$ can be measured at high speed, this technique is can be applied to real time monitor. When this technique for monitor of real time feed back control is used, an application to real time profiles control for $T_{e}$ and $n_{e}$ is possible.

\section{Acknowledgments}

The Authors are grateful to Mark Gwynneth for his technical assistance in initial test at ANU. This work is supported in part by Grant-in-Aid for Scientific Researches on Priority Area "Advanced diagnostics for burning plasma" from Ministry of Education, Culture, Sports, Science and Technology (No. 18035016).

[1] J. Sheffield, Plasma Scattering of Electromagnetic Radiation (Academic press, New York, 1975) p.191.

[2] K.D. Möller, Optics (University Science Books, Mill Valley, 1988) p.311.

[3] J. Howard, C. Michael, F. Glass and A. Cheetham, Rev. Sci. 
Instrum. 72, 888 (2001).

[4] J. Howard, Appl. Otp. 41, 197 (2002).

[5] J. Howard, C. Michael, F. Glass and A. Danielsson, Plasma Phys. Control. Fusion 45, 1143 (2003).

[6] J. Howard, Plasma Phys. Control. Fusion 48, 777 (2006).

[7] H. Koguchi, Y. Hirano, H. Sakakita et al., preprint of 21st IAEA Fusion Energy Conference, IAEA-CN-149/EX/P3-8 (2006).

[8] J. Howard, B. James and W. Smith, J. Phys. D: Appl. Phys.,
1435 (1979).

[9] T. Hatae, A. Nagashima, T. Kondoh et al., Rev. Sci. Instrum. 70, 772 (1999).

[10] T. Hatae, M. Nakatsuka and H. Yoshida, J. Plasma Fusion Res. 80, 870 (2004).

[11] T. Hatae, O. Naito, M. Nakatsuka and H. Yoshida, Rev. Sci. Instrum. 77, 10E508 (2006).

[12] H. Yoshida, O. Naito, O. Yamashita et al., Rev. Sci. Instrum. 70, 751 (1999). 